\title{
同手性金属有机框架(MOFs)维度的精准调控及苂光性质的研究
}

\author{
赵彦武* 李星张富强张祥 \\ (山西师范大学 化学与材料科学学院 磁性分子与磁信息材料教育部重点实验室 太原 030031)
}

\begin{abstract}
摘要 精准调控不同维度和拓扑的结构, 特别是具有同组分的手性结构代表了当今合成化学所面临的最具挑战性的问 题之一. 基于配位化学的不同维度杂化材料的自组装被认为是能够解决上述问题的一种有效途径. 本工作采用了已报 道的一维管(HMOF-2)和二维层(HMOF-3)合成所需的 $5,5^{\prime}-N, N$-二间苯二甲酸-( $\left.1 R, 2 R\right)$-环已烷二酰胺 $(R, R$-CHCAIP)配体 和锌盐 $\left(\mathrm{Zn}^{2+}\right)$, 在高温 $\left(100{ }^{\circ} \mathrm{C}\right)$ 溶剂热反应条件下自组装而成新的三维孔 MOFs (HMOF-4). 提出了辅助配体和反应温 度协同作用的策略, 进而精准调控获得了这三个结构多样化、维度不同的手性 MOFs, 在目前已报道的多维 MOFs 中, 这类具有同组分的多维手性 MOFs 是极为罕见的. 研究了其苂光性质, 用时间依赖的密度泛函理论(TDDFT)计算解释 了其发光机理. 它们发射光谱的微小差异说明 Zn-MOFs 的维度对荧光的影响较小. 这项工作为精准调控不同维度的同 手性 MOFs 提供了一个有特色的构筑方法.

关键词＼cjkstart精准调控; 同手性金属有机框架(MOFs); 维度; 协同作用; 菼光
\end{abstract}

\section{Precise Control of the Dimension of Homochiral Metal-Organic Frameworks (MOFs) and Their Luminescence Properties}

\author{
Yan-Wu Zhao* Xing Li Fu-Qiang Zhang Xiang Zhang \\ (Key Laboratory of Magnetic Molecules \& Magnetic Information Materials Ministry of Education, \\ School of Chemistry \& Material Science, Shanxi Normal University, Taiyuan 030031, China)
}

\begin{abstract}
To precisely control the construction of different dimensional and topological structure represents one of the most challenging issues facing synthetic chemistry nowadays, especially synthesizing different dimensions of chiral materials with identical compositions. Self-assembly of structural dimension of hybrid materials based on coordination chemistry has been regarded as an effective means addressed this issue. Herein, self-assembly of $\mathrm{Zn}$ salts with $R$, $R$-CHCAIP $\left(5,5^{\prime}-((1 R, 2 R)\right.$-cyclohexane dicarbonyl bis(azanediyl)) diisophthalic acid) generates a new 3D homochiral metal-organic framework $\mathrm{Zn}_{1.5}(R, R$-CHCAIP $)\left(\mathrm{H}_{2} \mathrm{O}\right)_{2}$ (HMOF-4) that uses the same ligand and metal as our reported 1D tube $\mathrm{Zn}_{2}\left(R, R\right.$-CHCAIP)Py $2\left(\mathrm{H}_{2} \mathrm{O}\right)$ (HMOF-2) and $2 \mathrm{D}$ layer $\left[\mathrm{Zn}_{4}(R, R \text {-CHCAIP) })_{2}\left(4,4^{\prime} \text {-bipy }\right)_{2}\left(\mathrm{H}_{2} \mathrm{O}\right)_{5}\right]^{-5} \mathrm{H}_{2} \mathrm{O} \cdot 3 \mathrm{DMA}$ (HMOF-3, DMA, $N, N$-dimethylacetamide) under the solvothermal reaction at high temperature $\left(100{ }^{\circ} \mathrm{C}\right)$. Based on a new strategy of the synergistic action of the auxiliary ligand and temperature, the structural diversity for HMOF-2, HMOF-3 and HMOF-4 from $1 \mathrm{D}$ to $2 \mathrm{D}$ and $3 \mathrm{D}$ is modulated during the assembly process, and the resulting multidimensional homochiral MOFs with identical composition are extremely rare in currently reported MOFs with different dimensions. The fluorescence of HMOF-4 and HMOF-2, HMOF-3 is investigated, and their fluorescent mechanisms are interpreted by time-dependent density functional theory (TDDFT) calculation. The tiny difference of their fluorescent emission spectra indicates the dimensions of $\mathrm{Zn}-\mathrm{MOF}$ have little effect on fluorescence. This work provides a distinctive construction means for precise control of the different dimension of homochiral MOFs.

Keywords precise control; homochiral metal-organic frameworks (MOFs); dimension; synergistic action; fluorescence
\end{abstract}

\section{1 引言}

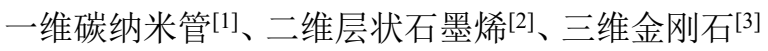
代表了三种不同维度和结构的碳材料, 由于其不同的性 质和功能而引起了人们广泛的研究兴趣. 目前, 一些热 门材料, 如钙钛矿 ${ }^{[4]}$ 和金属有机框架材料(metal-organic frameworks, MOFs) $)^{[5-7]}$ 也显示了类似于碳材料的这一特 点. 然而, 设计和合成这些不同维度和拓扑结构的材料
仍旧代表了当前合成化学中最具挑战性的任务之一. 基 于配位化学的多维杂化材料的合理设计和自组装是解 决该问题的一种有效途径 ${ }^{[8-10]}$. 近年来, 该领域的研究 主要集中在通过强的金属配位键或弱作用(如氢键和 $\pi-\pi$ 相互作用)自组装而成一维链或管、二维层和三维多孔 的 $\mathrm{MOFs}^{[11-12]}$.

不同维度的 MOFs 展现了不同的拓扑结构, 从而产

* E-mail: zhaoyw@sxnu.edu.cn

Received October 25, 2021; published October 27, 2021.

Supporting information for this article is available free of charge via the Internet at http://sioc-journal.cn.

Project supported by 1331 Project of Shanxi Province.

项目受山西省 1331 项目资助. 
生了不同的性质和功能 ${ }^{[5-7]}$. 一维金属有机纳米管的显 著特征是它拥有不同形状和尺寸的一维通道, 其一维的 限域空间可广泛应用于主客体化学 ${ }^{[13]}$. 与一维金属有 机纳米管不同，二维金属有机纳米片具有较大的比表面 积, 且其表面上拥有许多有效的活性位点, 这对分 离 ${ }^{[14]}$ 、传感和催化应用 ${ }^{[15]}$ 具有重要意义. 截至目前, MOFs 的研究主要集中在多孔的三维结构, 作为一种新 型的孔材料, 在气体吸附和储存、催化、传感、光学、 磁性和药物传输等领域表现出良好的性能 ${ }^{[16]}$. 在过去 的二十多年中, 已报道的一维、二维和三维 MOFs 主要 由不同的有机配体和金属 ${ }^{[6,17-19]}$ 或不同的有机配体和相 同的金属 ${ }^{[20-24]}$ 或不同的金属和相同的配体组成 ${ }^{[11,12,25-31]}$, 而由相同的有机配体和金属离子合成的一维、二维和三 维 MOFs 是有限的 ${ }^{[10,32-35]}$. 因为在合成 MOFs 时, 需要 考虑许多因素, 例如: 有机配体的柔性、配位点的数量 和方向、金属配位的可能性、金属离子和配体的物质的 量比、溶液的 $\mathrm{pH}$ 值、溶剂、温度、抗衡离子、辅助配 体等. 显然, 在确定了有机配体和金属这两种 MOFs 的 主要组分后, 仅通过改变反应条件来调控目标化合物的 维度和结构是具有挑战性的. 因此, 采用变换反应条件 来精准调控具有相同组分的多维 MOFs 的合成是一项更 有意义的研究. 迄今为止, 只有少数报道运用溶剂调 控 ${ }^{[32]} 、 \mathrm{pH}$ 调控 ${ }^{[33]}$ 、辅助配体作用 ${ }^{[10,36]}$ 组装而成多维 MOFs. 为了提高调控方法的可设计性和多样性, 有必 要开发更高效、更精准的合成策略. 而且, 目前由同一 有机配体和金属构建的一维、二维和三维 MOFs 主要是 非手性的 ${ }^{[10,32-35]}$, 而多维同手性 MOFs 则极为罕见. 鉴

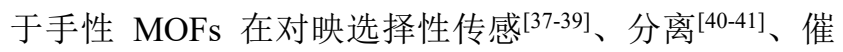
化 ${ }^{[15]}$ 等方面的广泛应用而变得越来越重要, 这促使我 们去开发其合理的合成方法及其应用研究.

在我们之前的研究中, 使用同手性配体 5,5'-N,N-二 间苯二甲酸- $(1 R, 2 R)$-环已烷二酰胺 $(R, R$-CHCAIP) 和锌 离子 $\left(\mathrm{Zn}^{2+}\right)$ 合成了一维管 $\mathrm{Zn}_{2}(R, R$-CHCAIP $) \mathrm{Py}_{2}\left(\mathrm{H}_{2} \mathrm{O}\right)$ (HMOF-2, Py, pyridine $)^{[38]}$ 和二维层 $\left[\mathrm{Zn}_{4}(R, R \text {-CHCAIP })_{2}-\right.$ $\left.\left(4,4^{\prime} \text {-bipy }\right)_{2}\left(\mathrm{H}_{2} \mathrm{O}\right)_{5}\right] \cdot 5 \mathrm{H}_{2} \mathrm{O} \cdot 3 \mathrm{DMA}$ (HMOF-3, DMA, $N, N$ dimethylacetamide $)^{[39]}$, 并研究了其传感应用. 在继续以 前的研究时, 我们尝试通过提高反应温度的方法, 用上 述的配体 $R, R$-CHCAIP 和 $\mathrm{Zn}$ 来设计和构筑了一种新的 三维同手性 MOF (HMOF-4). 因为较高的反应温度有利 于产生具有高配位数的 MOFs, 进而形成高维框架. 总 体上看来，这三个同手性 MOFs (homochiral MOFs, HMOFs)的合成体现了在辅助配体和温度协同作用下可 精准调控同手性 MOFs 维度的设计思想. 与通过控制单 一反应条件构筑多维 MOFs 的不确定性相比, 选择辅助 配体的加入是可预测的 (图 1). 实验证实了这三个 HMOFs 只有在辅助配体和温度的协同作用下才能成功 实现, 这是一种不同于单一条件调节的、可预测的设计 思想和精准调控维度的策略. 同时, 通过 TDDFT (time-dependent density functional theory)计算研究了它
们的发光机理，进一步证实了荧光的产生是由配体内电 荷转移引起的. 更为重要的是我们通过研究具有相同组 分不同维度和结构的 MOFs 苂光, 发现其发射光谱的差 异很小, 表明了这些 Zn-MOFs 的维度和结构对荧光影 响较小.

\section{2 结果与讨论}

\section{1 有机配体和同手性 MOFs 的合成}

用 $(1 R, 2 R)$-环己烷-1,2-二羧酸和 5-氨基间苯二甲酸 为原料合成了 $C 2$ 对称的手性配体 $R, R$-CHCAIP. 有趣的 是, $R, R$-CHCAIP 与硝酸锌在溶剂热反应下获得的配位 化合物呈现出不同维度的晶体结构(图 1). 与 HMOF-4 的合成比较, HMOF-2 的合成是通过选择末端辅助配体 吡啶, 在 $75{ }^{\circ} \mathrm{C}$ 下合成了一维金属有机纳米管; 以 4,4'联吡啶取代 HMOF-2 的吡啶, 可在 $85{ }^{\circ} \mathrm{C}$ 下得到二维类 波浪层 HMOF-3. 通过直接提高反应温度, 在 $100{ }^{\circ} \mathrm{C}$ 下 用 $R, R$-CHCAIP 和锌制备了三维多孔 HMOF-4. 值得注 意的是, 在辅助配体和反应温度的协同作用下, 获得了 一系列一维、二维和三维同手性 MOFs.

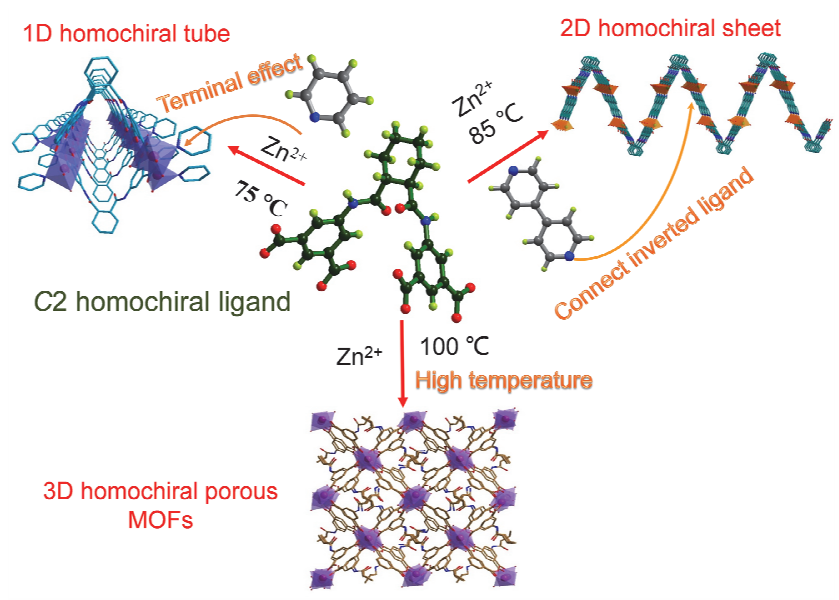

图 1 同手性的一维金属有机纳米管(HMOF-2)、二维类波浪层 (HMOF-3)和三维框架(HMOF-4)的构筑途径

Figure 1 The construction of homochiral 1D metal-organic nanotube (HMOF-2), 2D wave-like layer (HMOF-3) and 3D framework of HMOF-4

\subsection{HMOF-4 的结构描述}

$\mathrm{X}$ 射线单晶衍射数据分析表明 HMOF-4 属于四方 手性空间群 $P 4{ }_{1} 2{ }_{1} 2$ (表 $\mathrm{S} 1$, 见支持信息), 这与图 1 中的 一维管 HMOF-2 和二维类波浪层 HMOF-3 的单斜手性 空间群 $C 2$ 不同. 在 HMOF-4 的一个不对称单元中, 含 有 1.5 个 $\mathrm{Zn}(\mathrm{II})$ 离子、一个完整的 $R, R$-CHCAIP 配体、 一个配位水分子. 在 HMOF-4 晶体结构中, $\mathrm{Zn}(\mathrm{II})$ 离子存 在两种配位环境. 如图 $2 \mathrm{a}$ 所示, $\mathrm{Zn} 1$ 离子采用六配位模 式，位于扭曲的 $\left[\mathrm{ZnO}_{6}\right]$ 八面体中心，其中 “赤道” 平面 由三个配体中的三个羧酸氧原子和一个配位水的氧原 子组成. 而顶端位置则被一个配体的羧酸氧原子和另一 个水分子所占据. 四配位的 $\mathrm{Zn}^{2+}$ 显示了扭曲的 $\left[\mathrm{ZnO}_{4}\right]$ 四 
面体结构, 其顶点由四个配体中的四个羧酸氧原子占 据. 每个 $R, R$-CHCAIP 配体都具有 $\mu_{6}-\eta^{1} \eta^{1} \eta^{1} \eta^{1} \eta^{1} \eta^{1} \eta^{0} \eta^{0}$ 模 式, 连接了六个 $\mathrm{Zn}^{2+}$ (图 2c). $\mathrm{Zn}(\mathrm{II})$ 与氧原子之间的键长 为 $0.1977(2) \sim 0.2119(3) \mathrm{nm}$ (表 $\mathrm{S} 2$, 见支持信息), 围绕 $\mathrm{Zn}(\mathrm{II})$ 离子的键角为 $80.8(2)^{\circ} \sim 175.9(4)^{\circ}$ (表 S2). HMOF-4 的亚结构单元由三核簇 $\mathrm{Zn}_{2}(\mathrm{COO})_{4}\left(\mathrm{H}_{2} \mathrm{O}\right)_{2}$ 组成, 该三核 簇包含一个 $\mathrm{Zn} 1$ 离子、一个 $\mathrm{Zn} 2$ 离子、四个羧酸基团和 两个水分子(图 2d, 2e), 且 $\mathrm{Zn} 1-\mathrm{Zn} 2$ 距离为 0.3999(2) $\mathrm{nm}$. 每个簇连接了八个配体, 每个配体连接四个相邻的 $\mathrm{Zn}_{2}(\mathrm{COO})_{4}\left(\mathrm{H}_{2} \mathrm{O}\right)_{2}$ 亚结构单元, 进而形成了周期性的多 孔三维框架结构, 通过 PLATON 计算显示该 MOFs 的孔 隙率为 $21.7 \%$ (图 1). 从拓扑结构上看(图 2b), 如果将 $\mathrm{Zn}_{2}(\mathrm{COO})_{4}\left(\mathrm{H}_{2} \mathrm{O}\right)_{2}$ 亚结构单元看作是 8 连接的节点, $R, R$-CHCAIP 配体看作是 4 连接的连接子, 那么 HMOF-4 的结构可简化为 4,8-连接的网络, 并且通过 TOPOS 软件计算得到其点符号 (Schläfli) 是 $\left\{4^{12} \cdot 6^{12} \cdot 8^{4}\right\}\left\{4^{6}\right\} 2$ 以及拓扑类型为 flu (sqc169). a)

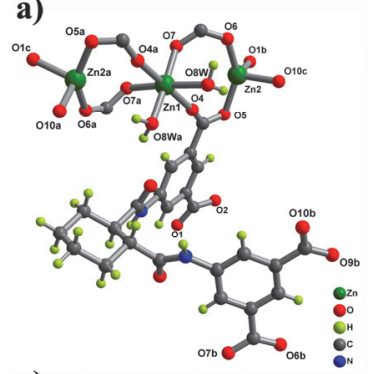

c)

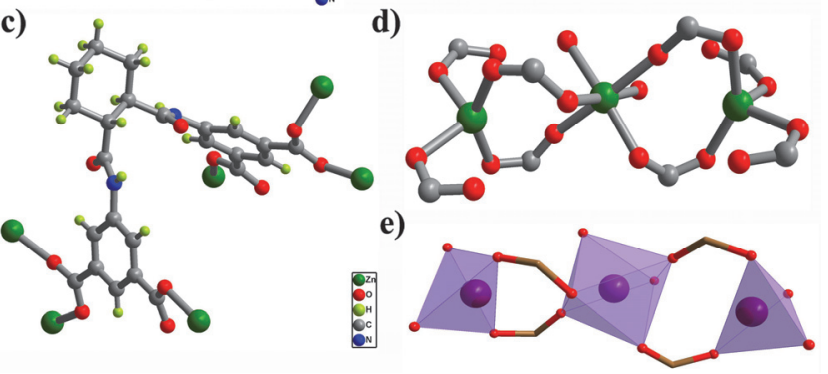

b)

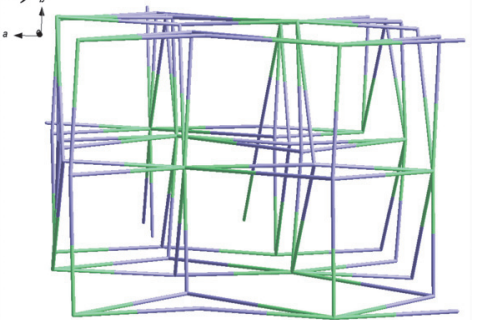

d)

e)
图 2 (a) HMOF-4 中 $\mathrm{Zn}$ 的配位环境图. 对称操作: a: $1-y, 1-x, 1 / 2$ $-z$; b: $1 / 2+x, 1 / 2-y, 3 / 4-z$; c: $-1 / 2+x, 3 / 2-y, 3 / 4-z$; (b) HMOF-4 的拓扑类型： flu (sqc169), (4,8)- 连接的网络 (亮绿色代表 $\mathrm{Zn}_{2}(\mathrm{COO})_{4}\left(\mathrm{H}_{2} \mathrm{O}\right)_{2}$ SBUs; 蓝紫色代表 $R, R$-CHCAIP 配体); (c) HMOF-4 中有机配体 $R, R$-CHCAIP 的配位模式; (d)和(e)含有八面体构型 $\mathrm{Zn} 1$ 和 四面体构型 $\mathrm{Zn} 2$ 的三核簇

Figure 2 (a) Representation of $\mathrm{Zn}$ coordination environment in HMOF-4. Symmetry operation: a: $1-y, 1-x, 1 / 2-z ; \mathrm{b}: 1 / 2+x, 1 / 2-y$, $3 / 4-z$; c: $-1 / 2+x, 3 / 2-y, 3 / 4-z$; (b) topological type: flu (sqc169), $(4,8)$-connected net in HMOF-4 (bright green: $\mathrm{Zn}_{2}(\mathrm{COO})_{4}\left(\mathrm{H}_{2} \mathrm{O}\right)_{2}$ SBUs, bluish violet: $R, R$-CHCAIP ligand); (c) coordination mode of organic ligand $R, R$-CHCAIP in HMOF-4; (d) and (e) trinuclear cluster with the coordination around $\mathrm{Zn} 1$ and $\mathrm{Zn} 2$, showing octahedral and tetrahedral geometries, respectively

\subsection{HMOF-4 与 HMOF-2 和 HMOF-3 的结构比较及其 维度的可设计性}

虽然一维 HMOF-2、二维 HMOF-3 和三维 HMOF-4 均由 $R, R$-CHCAIP 配体和 $\mathrm{Zn}$ 组成, 但这三个 HMOFs 结 构差异较大. 因它们的 $R, R$-CHCAIP 配体含有四个羧基,
故可作为 4 连接的连接子, 而作为节点的 $\mathrm{Zn}$ 族却表现 出不同的连接类型，这主要是由 $\mathrm{Zn}$ 离子的配位数及配 位模式不同引起的. 在 HMOF-2 和 HMOF-3 中, $\mathrm{Zn}$ 离子 都为四配位和五配位 (图 $\mathrm{S} 1$ ，见支持信息)，但在 HMOF-4 中存在高配位的 $\mathrm{Zn}$ (六配位) (图 2a). 与 HMOF-4 中的三核簇作为 8 连接节点相比, HMOF-2 中 的双核簇 $\mathrm{Zn}_{2}(\mathrm{COO})_{4}\left(\mathrm{H}_{2} \mathrm{O}\right) \mathrm{N}_{2}$ 作为 4 连接节点(图 $\mathrm{S} 1 \mathrm{c}$ 和 $\mathrm{S} 1 \mathrm{e})$, 该节点与 4 连接的连接子形成 $(4,4)$ 连接的一维螺 旋管(图 S1e), 同样, HMOF-3 的单核 $\mathrm{Zn}$ 族作为 3 连接节 点(图 S1d 和 S1f), 形成了 $(3,4)$ 连接的二维层(图 S1f). 很 显然，不同的 $\mathrm{Zn}$ 配位方式产生了不同的节点，进而导 致了结构的多样性.

三个 HMOFs 的结构和合成方案比较结果表明, 辅 助配体和温度对 MOFs 的维度调节起了重要作用. 在 HMOF-2 和 HMOF-3 中, 辅助配体的加入显示了框架的 可设计性和可预测性, 这主要表现为盖帽在 $\mathrm{Zn}$ 原子上 的辅助配体吡啶的末端效应阻止了其可能的进一步配 位，从而生成一维管 HMOF-2, 而二连接子 4,4'-联吡啶 连接了相邻的反向 $R, R$-CHCAIP 配体 (图 1), 从而形成 了二维类波浪层 HMOF-3. 显然温度的变化对 HMOF-2 和 HMOF-3 的形成是必要的, 因此它们显示了辅助配体 与温度的协同作用. 对于三维 HMOF-4 的生成, 则需要 提升更高的温度才能产生更高配位的 $\mathrm{Zn}$, 进而导致三 维框架的形成, 这符合三维 MOFs 的形成规律.

\subsection{HMOF-4 晶体的初步表征}

\subsubsection{HMOF-4 的 XPS 谱}

$X$ 射线光电子能谱(XPS)揭示了 HMOF-4 的元素组 成和化学状态. 如图 3a, 全谱图显示了 $\mathrm{C} 、 \mathrm{~N} 、 \mathrm{O}$ 和 $\mathrm{Zn}$ 的峰, 表明了 HMOF-4 的全部组分. Zn2p 谱中以 1021.7 $\mathrm{eV}$ 和 $1044.9 \mathrm{eV}$ 为中心的峰(图 3b)分别表示 $2 \mathrm{P} 3 / 2$ 和 $2 \mathrm{P} 1 / 2$ 两种电子轨道, 这应该归属于 $\mathrm{Zn}-\mathrm{O}-\mathrm{C}$ 键的形 成 ${ }^{[42]}$. 图 $3 \mathrm{c}$ 中的 $\mathrm{C} 1 \mathrm{~s}$ 谱显示了一个较宽的不对称峰, 由 284.7、286.3 和 $288.8 \mathrm{eV} 3$ 个峰组成, 分别对应于 $\mathrm{C}=$ $\mathrm{C} / \mathrm{C}-\mathrm{C}, \mathrm{C}-\mathrm{N}$ 和 $\mathrm{O}-\mathrm{C}=\mathrm{O}$ 键. $\mathrm{O} 1 \mathrm{~s}$ 信号分别在 531.8、 532.7 和 $533.4 \mathrm{eV}$ 处出峰(图 3d), 这三个峰分别归属于 $\mathrm{O}-\mathrm{C}=\mathrm{O} 、 \mathrm{O}=\mathrm{C}$ (酰胺羰基)和 $\mathrm{Zn}-\mathrm{O} . \mathrm{N} 1 \mathrm{~s}$ XPS 光谱可 裂分为两个峰, 分别位于 399.5 和 $398.9 \mathrm{eV}$, 对应于酰胺 的 $\mathrm{C}-\mathrm{N}$ 和 $\mathrm{N}-\mathrm{H}$ 基团(图 3e).

\subsubsection{HMOF-4 的粉末 X 射线衍射及红外光谱分析}

HMOF-4 晶体的粉末 X 射线衍射(PXRD)显示了其 模拟谱和测量谱的主峰基本吻合，表明了 HMOF-4 较纯 的物相(图 S2, 见支持信息). 从图 4 中 HMOF-4 的红外 光谱分析可知, $R, R$-CHCAIP 配体的羧基 $-\mathrm{COOH}$ 在 $1700 \mathrm{~cm}^{-1}$ 处显示了尖峰, 与之相比, HMOF-4 中羧基的 伸缩振动峰减小, 这主要是由羧酸氧与 $\mathrm{Zn}$ 形成配位键 引起的. 此外, 在 $482 \mathrm{~cm}^{-1}$ 处的 $\mathrm{Zn}-\mathrm{O}$ 峰也证实了在 HMOF-4 中 $\mathrm{Zn}$ 与羧酸氧原子形成了配位键. 

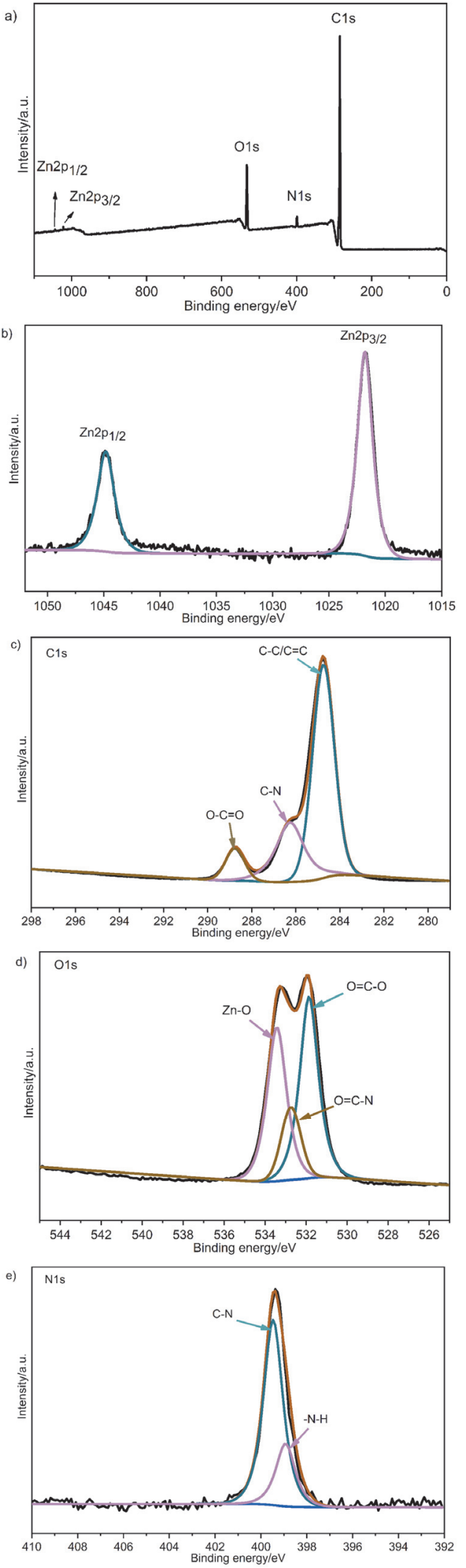

图 3 HMOF-4 的 XPS 谱: (a) 全谱, (b) Zn 2p, (c) C 1s, (d) O 1s 和(e) N $1 \mathrm{~s}$

Figure 3 XPS spectra of HMOF-4: (a) survey spectra, (b) Zn 2p, (c) C $1 \mathrm{~s}$, (d) $\mathrm{O} 1 \mathrm{~s}$ and (e) $\mathrm{N} 1 \mathrm{~s}$

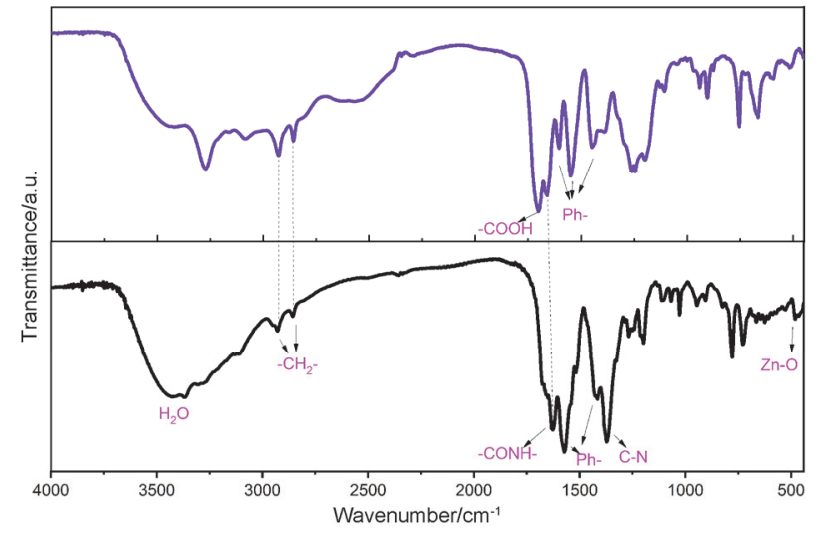

图 4 有机配体(紫色线)和 HMOF-4(黑色线)的红外光谱图

Figure 4 IR spectra of the organic ligand (violet line) and HMOF-4 (black line)

\subsubsection{HMOF-4 的热重分析(TGA)}

HMOF-4 晶体的 TGA 在 $30 \sim 800{ }^{\circ} \mathrm{C}$ 温度范围内进 行分析(图 5). TGA 曲线表明 HMOF-4 在空气中具有较 高的热稳定性. 连续三次失重分别是客体水分子、配位 水分子和客体的 $N, N$-二甲基甲酰胺(DMF) 分子. 有机 配体的分解发生在 $340 \sim 500{ }^{\circ} \mathrm{C}, 500{ }^{\circ} \mathrm{C}$ 后骨架完全坍 塌, 最终的残留物为氧化锌.

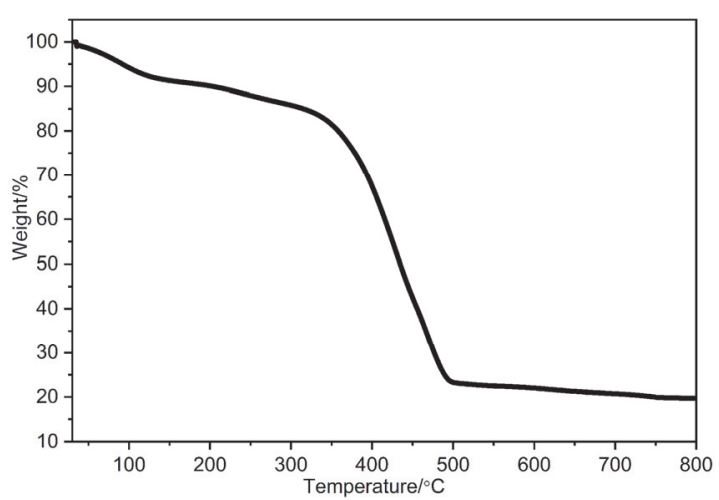

图 5 HMOF-4 的 TGA 曲线

Figure 5 TGA curve of HMOF-4

\subsection{4 有机配体和 HMOF-4 的圆二色谱(CD)分析}

HMOF-2、HMOF-3 和 HMOF-4 在乙腈中的 CD 光 谱分别在 226、228 和 $229 \mathrm{~nm}$ 附近显示了正的科顿效应, 而在 260、276 和 $257 \mathrm{~nm}$ 附近显示了负的科顿效应(图 S3，见支持信息)，很明显，三个不同维度的手性 MOFs 呈现了不同的 $\mathrm{CD}$ 信号, 这可能是与其晶体结构有关. 与 $R, R$-CHCAIP(图 S3a)相比, 这三个晶体的 $\mathrm{CD}$ 信号向 长波长方向有较大的位移, 这可能是由自由配体的点手 性向 HMOF-4 聚合物手性的 “繁殖” 引起的.

\subsection{HMOF-4 的苂光、机理研究以及与 HMOF-2 和 HMOF-3 的荧光差异}

HMOF-4 晶体在 $469 \mathrm{~nm}$ 附近显示了最大的苂光发 射峰, 在 $449 \mathrm{~nm}$ 处有较小的肩峰 (图 6a), 这和 
HMOF-3 出现在 $472 \mathrm{~nm}$ 最大的苂光发射峰和 $450 \mathrm{~nm}$ 处 较小的肩峰是类似的 (图 $6 \mathrm{~b}$ ). 与固态的配体 $R, R$-CHCAIP 在 $470 \mathrm{~nm}$ 处的最大发射峰相比(图 $\mathrm{S} 4$, 见 支持信息), HMOF-2 和 HMOF-4 表现出轻微的蓝移, 这 与 HMOF-3 在 $472 \mathrm{~nm}$ 处的最大发射峰轻微的红移不同 (图 6b).
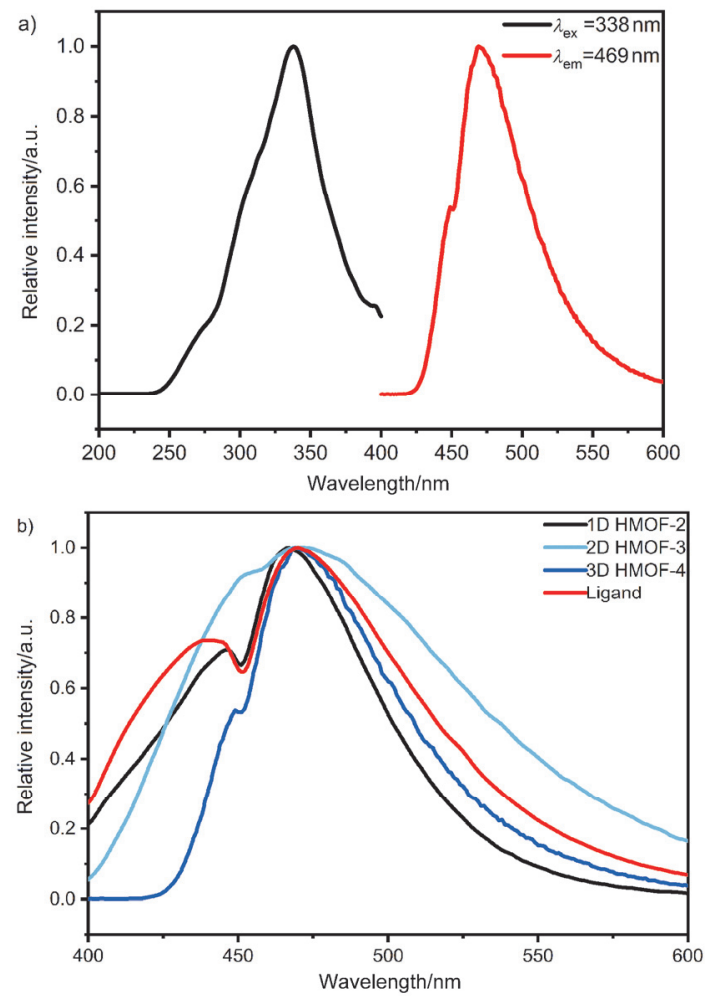

图 6 (a) 固态的 HMOF-4 的荧光激发谱和发射谱; (b) 固态的有机配 体 $R, R$-CHCAIP、HMOF-2、HMOF-3 和 HMOF-4 的荧光发射光谱 Figure 6 (a) Excitation and emission spectra of solid HMOF-4; (b) emission spectra of solid $R, R$-CHCAIP ligand, HMOF-2, HMOF-3 and HMOF-4

为了全面地理解三种 HMOFs 的光致发光性质, 有 必要深入研究它们的电子结构, 因为材料的光致发光性 质主要依赖于它们的电子结构. 而电子结构取决于化学 成分和晶体结构. 在以往的研究中, 不同维度 MOFs 的 荧光发射主要归因于化学成分, 包括金属中心发射、配 体中心发射、客体中心发射以及成分的电荷转移(包括 配体到金属的电荷转移(LMCT)、金属到配体的电荷转 移(MLCT)、配体到配体的电荷转移(LLCT)和主客体电 荷转移) ${ }^{[17,21-22,25,33]}$, 而很少研究与晶体结构(配位环境、 构型畸变等)的关系 ${ }^{[23,28]}$. 而且, 大多数已报道的多维 MOFs 的发光机理都是从电子的吸收光谱和发射光谱中 推测获得, 尚未得到证实, 我们认为这些推测非常有必 要通过理论计算加以证实. 在以前的研究中我们已经证 实了 HMOF-2 和 HMOF-3 主要是以配体为中心的发光, 在本研究中, 我们将继续利用前线分子轨道(FMOs)理 论研究与它们发光相关的电荷转移.
对优化后的配体、HMOF-2 和 HMOF-3 模型的几何 结构进行了 TDDFT 计算(图 S5, 见支持信息), 以解释 这些 HMOFs 中电子跃迁的机理, 光谱数据如表 S3( 见支 持信息)所示. 由图 7 可以看出, HMOF-2 的最高占据分 子轨道(HOMO)和最低未占据分子轨道( LUMO)的电子 密度分布分别位于 $R, R$-CHCAIP 配体的 $\mathrm{PhNH}-\mathrm{C}=\mathrm{O}$ 片段和吡啶辅助配体上 (图 7b). 同样, HMOF-3 的 HOMO 和 LUMO 电子密度各自分布在 $R, R$-CHCAIP 的 $\mathrm{PhNH}-\mathrm{C}=\mathrm{O}$ 片段和 4,4'-联吡啶上(图 7c). 鉴于 HMOF-4 结构的复杂性及成分中配体作为苂光的主要 贡献者, 我们截取了 HMOF-4 的配体片段作为 TDDFT 计算模型, 如图 7a 所示, 配体的 HOMO 和 LUMO 电子 密度分别分布在完整配体的两个 $\mathrm{PhNH}-\mathrm{C}=\mathrm{O}$ 片段上. 整体上，图 7 展示了配体和 MOFs 的不同分子轨道在最 低吸收能带中的参与状况. 在所有情况下, 由于锌几乎 没有贡献, 因此从 HOMO 到 LUMO 的跃迁都属于配体 内的电荷转移. 表 S3 中两种 HMOFs 和配体的光谱数据 显示了光谱实验结果与计算结果能较好地匹配. 此外, 相比配体而言, 由于 HMOF-2 和 HMOF-4 较为刚性的结 构，抑制了非辐射衰减过程，可能导致了波长蓝移，而 在 HMOF-3 中, 层与层之间的 $\pi-\pi 、 \mathrm{C}-\mathrm{H} \cdots \pi$ 和 $\mathrm{C}-\mathrm{O} \cdots$ $\pi$ 等堆积作用导致了能隙值更低 ${ }^{[39]}$, 可能引起了波长轻 微的红移, 同时纳米层相对柔性的结构可能使其苂光发 射光谱变宽. 更为重要的是, 我们从 HMOF-2、HMOF-3 和 HMOF-4 的微小荧光差异中可得出这些 Zn-MOFs 的 维度和结构对荧光影响较小.

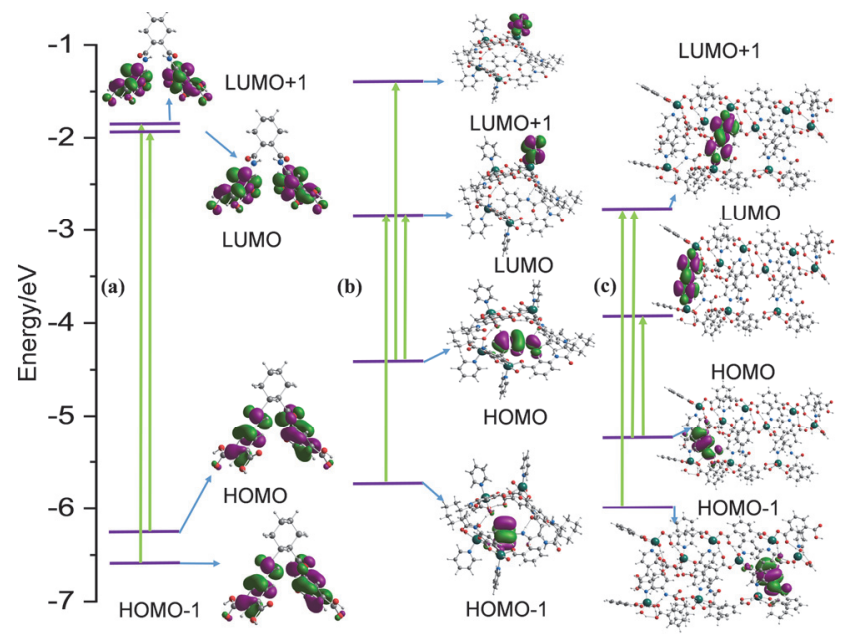

图 7 描述由(a)有机配体, (b) HMOF-2 和(c) HMOF-3 的最低能量吸收 带组成的主要跃迁能级图

Figure 7 Energy-level diagram depicting the dominant transitions that comprise the lowest-energy absorption bands for (a) organic ligand, (b) HMOF-2 and (c) HMOF-3

\section{3 结论}

总之, 将一种新型三维多孔 HMOF-4 与我们之前报 道的一维管 HMOF-2 和二维层 HMOF-3 结合，展示了一 项完整而连续的精准调控同手性 MOFs 维度的研究. 这 
三种 HMOFs 都是在辅助配体和温度的协同作用下，用 相同的有机配体 $5,5^{\prime}-N, N$-二间苯二甲酸- $(1 R, 2 R)$-环己烷 二酰胺 $(R, R-\mathrm{CHCAIP})$ 和金属 $\mathrm{Zn}$ 离子合成的. 根据所加 入辅助配体的特点, 不同维度的同手性 MOFs 是可设计 和可预测的, 如一维 HMOF-2 中辅助配体吡啶的末端效 应和 HMOF-3 中二连接的 4,4'-联吡啶辅助配体导致了 二维波状层的形成. 高温下生成的高配位结构(三维 HMOF-4 含六配位的 Zn), 进而形成高维框架, 实验证 实了该设计思路是可行的. 总的说来, 在目前已报道的 一系列不同维度的非手性 MOFs 中, 这是一例罕见的同 手性一维、二维和三维的 MOFs 的合成. 此外, 一维 HMOF-2、二维 HMOF-3 和三维 HMOF-4 的同手性 MOFs 均具有与有机配体相似的荧光发射, 且荧光最大 发射波长位于 $470 \mathrm{~nm}$ 左右, 通过 TDDFT 计算解释了苂 光机理, 可归因于配体内的电荷转移. 而且从三个维度 MOFs 的微小苂光差异中可得出这几个 Zn-MOFs 的维 度和结构对荧光影响较小的结论. 本工作可作为用同一 配体和金属精准调控不同维度 MOFs 合成的一个例子. 未来, 研究并开发不同维度的 MOFs, 进一步探索其磁 性、电化学和光催化等性能是一个值得关注的方向.

\section{4 实验部分}

$\mathrm{Zn}_{1.5}(R, R-\mathrm{CHCAIP})\left(\mathrm{H}_{2} \mathrm{O}\right)_{2}$ (HMOF-4) 的合成: 将 $\mathrm{Zn}\left(\mathrm{NO}_{3}\right)_{2} \cdot 6 \mathrm{H}_{2} \mathrm{O}(53 \mathrm{mg}, 0.18 \mathrm{mmol}) 、 R, R$-CHCAIP (29 $\mathrm{mg}, 0.058 \mathrm{mmol}) 、$ 混合溶剂 $\mathrm{DMF}$ 与去离子水 $(3 \mathrm{~mL} / 3$ $\mathrm{mL})$ 的混合物放入盖帽的玻璃小瓶中. 混合溶液剧烈摚 拌 $20 \mathrm{~min}$ 后, 在 $100{ }^{\circ} \mathrm{C}$ 下加热 $72 \mathrm{~h}$, 得到无色棒状晶体, 缓慢冷却至室温, 然后过滤, 用 DMF 洗涤, 在空气气氛 下干燥, 获得了基于 $R, R$-CHCAIP 配体 $35 \%$ 的产率. Anal. calcd for $\mathrm{C}_{24} \mathrm{H}_{20} \mathrm{~N}_{2} \mathrm{O}_{11} \mathrm{Zn}_{1.5}$ : C 47.22, H 3.30, N 4.59; found C 47.16, H 3.42, N 4.56. IR (KBr) v: 3429 (vs), 2928 (w), 2854 (w), 1629 (s), 1580 (s), 1415 (s), 1375 (s), $1198(\mathrm{w}), 825(\mathrm{~s}), 773(\mathrm{w}), 482(\mathrm{~s}) \mathrm{cm}^{-1}$.

HMOF-4 的 CCDC 号是 2102097, 这包含了本文中 补充的晶体数据.

\section{References}

[1] Schroeder, V.; Savagatrup, S.; He, M.; Lin, S.; Swager, T. M. Chem. Rev. 2019, 119, 599.

[2] Liu, Y.; Dong, X.; Chen, P. Chem. Soc. Rev. 2012, 41, 2283.

[3] Forcolin, G. T.; Grilj, V.; Hamilton, B.; Li, L.; McGowan, M.; Murphy, S. A.; Oh, A.; Skukan, N.; Whitehead, D.; Zadoroshnyj, A. Diam. Relat. Mater. 2016, 65, 75.

[4] Tyagi, P.; Arveson, S. M.; Tisdale, W. A. J. Phys. Chem. Lett. 2015, $6,1911$.

[5] Babu, R.; Roshan, R.; Gim, Y.; Jang, Y. H.; Kurisingal, J. F.; Kim, D. W.; Park, D.-W. J. Mater. Chem. A 2017, 5, 15961.

[6] Zhang, Q.; Shreeve, J. M. Angew. Chem. Int. Ed. 2014, 53, 2540.

[7] Cao, L.; Lin, Z.; Shi, W.; Wang, Z.; Zhang, C.; Hu, X.; Wang, C.; Lin, W. J. Am. Chem. Soc. 2017, 139, 7020.
[8] Kitagawa, S.; Kitaura, R.; Noro, S. Angew. Chem. Int. Ed. 2004, 43, 2334.

[9] Moreno, J. M.; Velty, A.; Díaz, U.; Corma, A. Chem. Sci. 2019, 10, 2053.

[10] Pan, J.; Ma, Y.-J.; Han, S.-D.; Xue, Z.-Z.; Wang, G.-M. Cryst. Growth Des. 2019, 19, 2919.

[11] Sun, L.; Li, G.-Z.; Xu, M.-H.; Li, X.-J.; Li, J.-R.; Deng, H. Eur. J. Inorg. Chem. 2012, 2012, 1764.

[12] Haldar, R.; Gurunatha, K. L.; Sikdar, N.; Maji, T. K. Inorg. Chem. Front. 2015, 2, 278.

[13] Xin, X.; Zhang, M.; Zhao, J.; Han, C.; Liu, X.; Xiao, Z.; Zhang, L.; Xu, B.; Guo, W.; Wang, R.; Sun, D. J. Mater. Chem. C 2017, 5, 601.

[14] Peng, Y.; Li, Y.; Ban, Y.; Jin, H.; Jiao, W.; Liu, X.; Yang, W. Science 2014, 346, 1356.

[15] Tan, C.; Yang, K.; Dong, J.; Liu, Y.; Liu, Y.; Jiang, J.; Cui, Y. J. Am. Chem. Soc. 2019, 141, 17685.

[16] Zang, Y.; Li, L.-K.; Zang, S.-Q. Coord. Chem. Rev. 2021, 440, 213955.

[17] Zhang, L.-P.; Ma, J.-F.; Yang, J.; Liu, Y.-Y.; Wei, G.-H. Cryst. Growth Des. 2009, 9, 4660.

[18] He, H.; Collins, D.; Dai, F.; Zhao, X.; Zhang, G.; Ma, H.; Sun, D. Cryst. Growth Des. 2010, 10, 895.

[19] Hu, B.; Wang, Y.-G.; Dai, Y.; Peng, Y.-X.; Huang, W. Polyhedron 2014, 83, 205.

[20] Abu-Youssef, M. A. M.; Langer, V. Polyhedron 2006, 25, 1187.

[21] Ren, P.; Liu, M.-L.; Zhang, J.; Shi, W.; Cheng, P.; Liao, D.-Z.; Yan, S.-P. Dalton Trans. 2008, 4711.

[22] Zhou, Y.-X.; Shen, X.-Q.; Du, C.-X.; Wu, B.-L.; Zhang, H.-Y. Eur. J. Inorg. Chem. 2008, 2008, 4280.

[23] Niu, C.-Y.; Zheng, X.-F.; He, Y.; Feng, Z.-Q.; Kou, C.-H. CrystEngComm 2010, 12, 2847.

[24] Sun, D.; Zhang, N.; Xu, Q.-J.; Wei, Z.-H.; Huang, R.-B.; Zheng, L.-S. Inorg. Chim. Acta 2011, 368, 67.

[25] Wen, L.-L.; Dang, D.-B.; Duan, C.-Y.; Li, Y.-Z.; Tian, Z.-F.; Meng, Q.-J. Inorg. Chem. 2005, 44, 7161.

[26] Colodrero, R. M. P.; Olivera-Pastor, P.; Cabeza, A.; Papadaki, M.; Demadis, K. D.; Aranda, M. A. G. Inorg. Chem. 2010, 49, 761

[27] Pachfule, P.; Panda, T.; Dey, C.; Banerjee, R. CrystEngComm 2010, $12,2381$.

[28] Zhang, W. H.; Dong, Z.; Wang, Y. Y.; Hou, L.; Jin, J. C.; Huang, W. H.; Shi, Q. Z. Dalton Trans. 2011, 40, 2509.

[29] Dong, W.-W.; Li, D.-S.; Zhao, J.; Wu, Y.-P.; Meng, C.-X.; Wang, Y.-Y. Inorg. Chim. Acta 2012, 384, 260.

[30] Yang, H.-X.; Liang, Z.; Hao, B.-L.; Meng, X.-R. J. Solid State Chem. 2014, 218, 23.

[31] Cui, L.-S.; Meng, X.-M.; Li, Y.-G.; Huang, K.-R.; Li, Y.-C.; Long, J.-Q.; Yao, P.-F. CrystEngComm 2019, 21, 3798.

[32] Chen, S.-C.; Zhang, Z.-H.; Huang, K.-L.; Chen, Q.; He, M.-Y.; Cui, A.-J.; Li, C.; Liu, Q.; Du, M. Cryst. Growth Des. 2008, 8, 3437.

[33] Gao, Q.; Jiang, F.-L.; Wu, M.-Y.; Huang, Y.-G.; Yuan, D.-Q.; Wei, W.; Hong, M.-C. CrystEngComm 2009, 11, 918.

[34] Cao, Y.; Zhu, Z.; Xu, J.; Wang, L.; Sun, J.; Chen, X.; Fan, Y. Dalton Trans. 2015, 44, 1942.

[35] Lucenti, E.; Cariati, E.; Previtali, A.; Marinotto, D.; Forni, A.; Bold, V.; Kravtsov, V. C.; Fonari, M. S.; Galli, S.; Carlucci, L. Cryst Growth Des. 2019, 19, 1567.

[36] Singh, D.; Nagaraja, C. M. Cryst. Growth Des. 2015, 15, 3356.

[37] Wanderley, M. M.; Wang, C.; Wu, C.-D.; Lin, W. J. Am. Chem. Soc. 2012, 134, 9050 .

[38] Zhao, Y.-W.; Zhang, X.-M. J. Mater. Chem. C 2020, 8, 4453.

[39] Zhao, Y.-W.; Guo, L.-E.; Zhang, F.-Q.; Yao, J.; Zhang, X.-M. ACS Appl. Mater. Interfaces 2021, 13, 20821.

[40] Guo, J.; Zhang, Y.; Zhu, Y.; Long, C.; Zhao, M.; He, M.; Zhang, X.; Lv, J.; Han, B.; Tang, Z. Angew. Chem. Int. Ed. 2018, 57, 6873.

[41] Hu, C.; Li, L.; Yang, N.; Zhang, Z.; Xie, S.; Yuan, L. Acta Chim. Sinica 2016, 74, 819 (in Chinese). (胡聪, 李丽, 杨娜, 张紫恒, 谢 生明，袁黎明，化学学报, 2016, 74, 819.)

[42] Kim, H. H.; Lee, Y.; Lee, Y. J.; Jeong, J.; Yi, Y.; Park, C.; Yim, S.-Y.; Angadi, B.; Ko, K.-J.; Kang, J.-W.; Choi, W. K. ACS Photonics 2020, 7,723 . 